\title{
Hadron Masses and Quark Condensate from Overlap Fermions
}

\author{
K. F. Liu ${ }^{\mathrm{a} b}{ }^{*}$, S. J. Dong ${ }^{\mathrm{b}}$, F. X. Lee ${ }^{\mathrm{c}} \mathrm{d}$, and J. B. Zhang ${ }^{\mathrm{b}} \mathrm{c}$ e \\ ${ }^{a}$ National Center for Theoretical Sciences, P.O. Box 2-131, Hsinchu, Taiwan \\ ${ }^{\text {b}}$ Department of Physics and Astronomy, University of Kentucky, Lexington, KY 40506, USA \\ ${ }^{\text {c} D e p a r t m e n t ~ o f ~ P h y s i c s, ~ G e o r g e ~ W a s h i n g t o n ~ U n i v e r s i t y, ~ W a s h i n g t o n, ~ D C ~ 20052, ~ U S A ~}$ \\ dJefferson Lab, 12000 Jefferson Avenue, Newport News, VA 23606, USA \\ eZhejiang Institute of Modern Physics, Zhejiang University, Hangzhou 310027, P.R. China
}

We present results on hadron masses and quark condensate from Neuberger's overlap fermion. The scaling and chiral properties and finite volume effects from this new Dirac operator are studied. We find that the generalized Gell-Mann-Oakes-Renner relation is well satisfied down to the physical $\mathrm{u}$ and $\mathrm{d}$ quark mass range. We find that in the range of the lattice spacing we consider, the $\pi$ and $\rho$ masses at a fixed $m_{\pi} / m_{\rho}$ ratio have weak $O\left(a^{2}\right)$ dependence.

The recent advance in chiral fermion formulation which satisfies Ginsparg-Wilson relation has a great promise in implementing chiral fermion for lattice QCD at finite lattice spacing. It is shown to have exact chiral symmetry on the lattice [1] and it has no order $a$ artifacts [2]. Neuberger's Dirac operator [3] derived from the overlap formalism has a compact form in four dimensions which involves a matrix sign function

$D=\frac{1}{2}\left[1+\mu+(1-\mu) \gamma_{5} \epsilon(H)\right]$

In this talk, I present some preliminary results from our numerical implementation of the Neuberger fermion. We adopt the optimal rational approximation of the matrix sign function [4] with 12 terms in the polynomials. The smallest 10 to 20 eigenvalues of $H^{2}$ are projected out for exact evaluation of the sign function for these eigenstates. We use multi-mass conjugate gradient as the matrix solver for both the inner and outer loops. With residuals at $10^{-7}$, the inner loop takes $\sim 200$ iterations and the outer loop takes $\sim 100$ iterations. We check the unitarity of the matrix $V=\gamma_{5} \epsilon(H)$. For $V x=b$, we find $\left|x^{\dagger} x-b^{\dagger} b\right| \sim 10^{-9}$. Even for topological sectors

\footnotetext{
${ }^{*}$ Talk presented at Lattice '99, Pisa, Italy
}

with $Q \neq 0$, we find the critical slowing down is much milder than that of the Wilson fermion and there are no exceptional configurations. The critical slowing down sets in quite abruptly after $\mu a=0.003$ which is already at the physical $\mathrm{u}$ and $\mathrm{d}$ masses.

It is shown [5] that the generalized Gell-MannOakes-Renner (GOR) relation

$\mu \int d^{4} x\langle\pi(x) \pi(0)\rangle=2\langle\bar{\Psi} \Psi\rangle$

with $\pi(x)$ being the pion interpolation field, is satisfied for each quark mass and volume, configuration by configuration. We utilize this relation as a check of our numerical implementation of the Neuberger operator. We find that for the lattices we considered $\left(6^{3} \times 12, \beta=5.7,5.85 ; 8^{3} \times 16, \beta=\right.$ 5.85 , and $\left.10^{3} \times 20, \beta=5.85,6.0\right)$ the GOR relation is satisfied very well (to within $1 \%$ ) all the way down to the smallest mass $\mu a=0.0001$ for the $Q=0$ sector. For the $Q \neq 0$ sector, the presence of zero modes demands higher precision for the approximation of $\epsilon(H)$. For example, we show in Fig. 1 the ratio of the right to left side of Eq. (2) for a configuration with topology on the $6^{3} \times 12$ lattice at $\beta=5.7$ as a function of the quark mass. When only 10 smallest eigenmodes are projected, we see that the ratio deviates from 
one for small quark masses. The situation is considerably improved when 20 smallest eigenmodes are included. The situation is better than the domain-wall fermion case when the size of the fifth dimension is limited to $L_{s}=10$ to 48 [6].

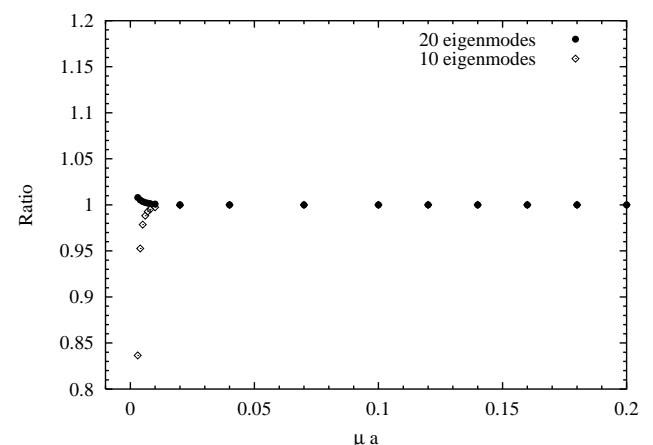

Figure 1. Ratio of the right to left side of Eq. (2) for a configuration with topology. $\diamond / \bullet$ indicates the case with projection of $10 / 20$ smallest eigenmodes.

We also calculate the quark condensate $\langle\bar{\Psi} \Psi\rangle$ with 3 to $6 Z_{2}$ noises for each configuration. For small quark mass, it has the form

$\langle\bar{\Psi} \Psi\rangle=\frac{\langle|Q|\rangle}{\mu V}+c_{0}+c_{1} \mu$.

The singular term which is due to the zero modes in the configurations with topology $(Q \neq 0)$ is specific to the quenched approximation. It will be suppressed when the determinant is included in the dynamical fermion case. We see this clearly in the following figure which is first seen with the domain-wall fermion [6]. A fit to the formula in Eq. (3) is given in Fig. 2. We see that $c_{0}$ is nonzero. The standard definition of the quark chiral condensate entails the extrapolation of $c_{0}$ to the infinite volume before taking the massless limit. Another way is to consider the finite-size scaling [7]. When the size of the lattice is much smaller than the pion Compton wavelength, i. e. $L \ll$ $1 / m_{\pi}$, the $\langle\bar{\Psi} \Psi\rangle$ is proportional to $\mu \Sigma^{2} V$ for small masses besides the $\frac{\langle|Q|\rangle}{\mu V}$ term due to quenching. From this, the infinite volume condensate $\Sigma$ can be extracted. We plot in Fig. $3\langle\bar{\Psi} \Psi\rangle a^{3} / \mu a$ vs $\mu a$ in the $Q=0$ sector for 3 lattice volumes $\left(6^{3} \times\right.$ $12,8^{3} \times 16$, and $\left.10^{3} \times 20\right)$ at $\beta=5.85$. We see

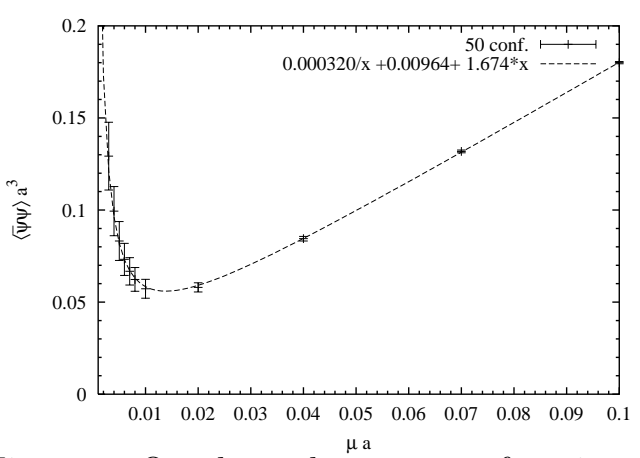

Figure 2. Quark condensate as a function of the quark mass. This is done on $506^{3} \times 12$ lattices at $\beta=5.7$.

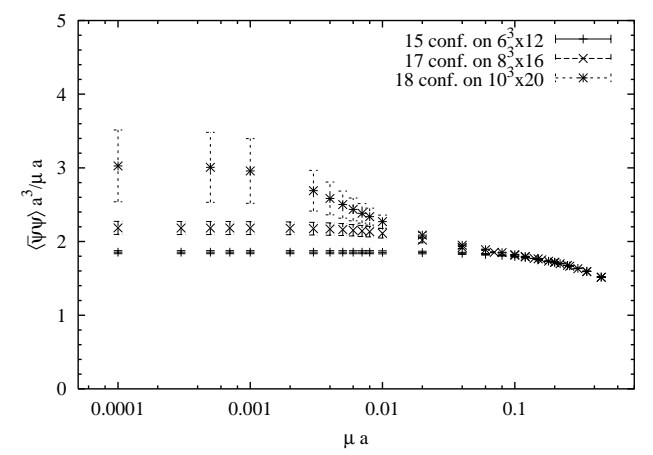

Figure 3. $\langle\bar{\Psi} \Psi\rangle a^{3} / \mu a$ vs $\mu a$ in the $Q=0$ sector for 3 lattice volumes at $\beta=5.85$.

that they are quite flat which indicates that the condensate is indeed proportional to $\mu$ and we also see that they increase with volume.

We have calculated the $\pi, \rho$ and nucleon masses. A typical result on the $8^{3} \times 16$ lattice at $\beta=5.85$ is given in Fig. 4. We see the finite volume effect on the nucleon mass when $\mu a$ is smaller than $\sim 0.15$. To see the behavior of pion masses near the chiral limit, we plot $m_{\pi}^{2} a^{2}$ as a function of $\mu a$ in Fig. 5 for three lattices with about the same physical volume. It appears that there might be a $\sqrt{\mu a}$ behavior in the very small $\mu a$ region which we will explore further. When we project only 10 smallest eigenmodes in the approximation for the sign function in the $6^{3} \times 12$ case, we see that $m_{\pi}^{2} a^{2}$ tends to a finite value as $\mu a \rightarrow 0$. This implies a residual mass due to the poor approximation of $\epsilon(H)$, a behavior similar to that observed in the domain-wall fermion with 


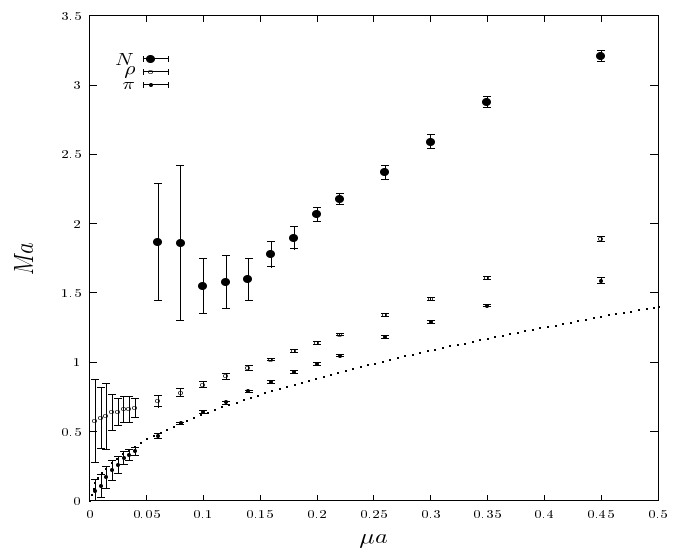

Figure 4. Masses of $\pi, \rho$ and $N$ on the $8^{3} \times 16$ lattice at $\beta=5.85$ are plotted vs $\mu a$.

finite $L_{s}$. Finally, we check scaling. We plot in Fig. $6 \pi / \sqrt{\sigma}$ and $\rho / \sqrt{\sigma}$ vs $\sigma a^{2}$ where $\sigma$ is the string tension from which the lattice spacings are determined [8]. It is known that the overlap operator does not have $O(a)$ artifacts. Now it appears that the $O\left(a^{2}\right)$ errors are small.

This work is partially supported by DOE Grants DE-FG05-84ER40154 and DE-FG0295ER40907. We thank R. Edwards for sharing his experience in implementing the sign function solver. We also thank H. Neuberger for stimulating discussions.

\section{REFERENCES}

1. M. Lüscher, Phys. Lett. B 428, 342 (1998); T. W. Chiu and S. V. Zenkin, Phys. Rev. D 59, 074501 (1999).

2. F. Niedermayer, Nucl. Phys. B 73(Proc. Suppl.), 105 (1999).

3. H. Neuberger, Phys. Lett. B 417, 141 (1998).

4. R. G. Edwards, U. M. Heller and R. Narayanan, Nucl. Phys. B 540, 457 (1999).

5. R. G. Edwards, U. M. Heller and R. Narayanan, Phys. Rev. D 59, 094510 (1999).

6. P. Chen et al., Nucl. Phys. B 73 (Proc. Suppl.), 207 (1999).

7. P. Hernádez, K. Jansen, and L. Lellouch, heplat/9907022.

8. T. Yoshie, Nucl. Phys. B 63(Proc. Suppl.), 3 (1998).
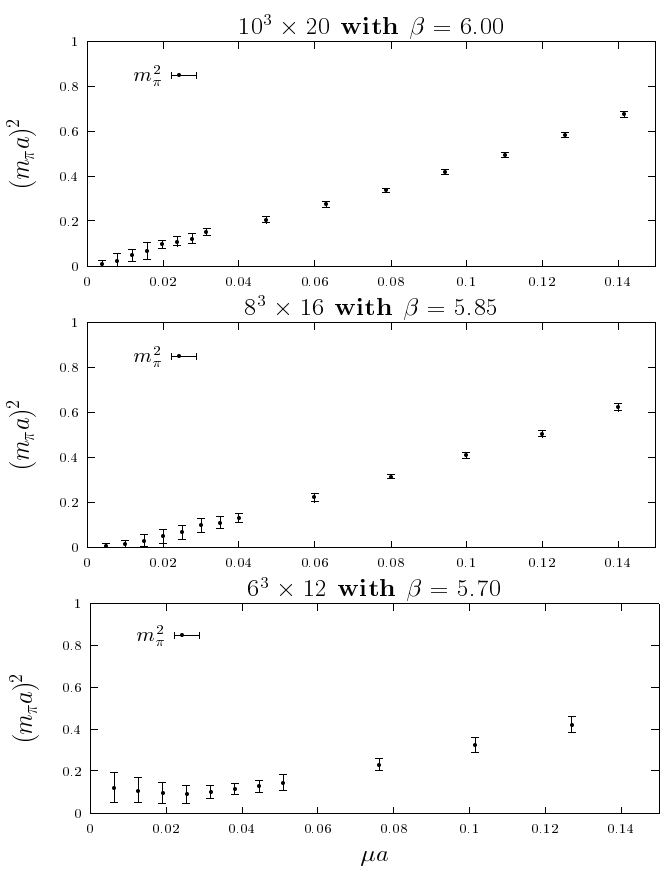

Figure 5. $m_{\pi}^{2} a^{2}$ are plotted as a function of $\mu a$ for three lattices with about the same physical volume.
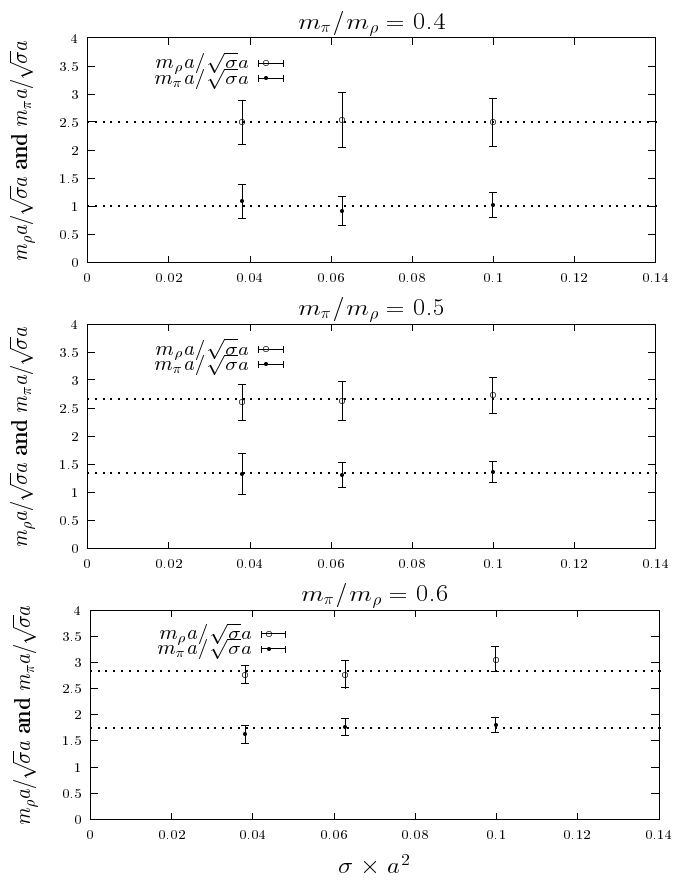

Figure 6. $\pi / \sqrt{\sigma}$ and $\rho / \sqrt{\sigma}$ are plotted vs $\sigma a^{2}$ for three lattices with similar physical volumes. 